\title{
3D MHD modelling of low current - high voltage DC plasma torch under restrike mode
}

\author{
A Lebouvier ${ }^{1,2}$, C Delalondre ${ }^{3}$, F Fresnet ${ }^{2}$, F Cauneau ${ }^{1}$ and L Fulcheri ${ }^{1,4}$ \\ ${ }^{1}$ Center for Energy and Processes - MINES ParisTech, Rue Claude Daunesse, 06904 \\ Sophia Antipolis, France \\ ${ }^{2}$ Technocentre Renault, DREAM/DELT'A, 78288 Guyancourt, France. \\ ${ }^{3}$ EDF R\&D, MFEE Department, 6 quai Watier, 78400 Chatou, France.
}

E-mail: laurent.fulcheri@mines-paristech.fr

\begin{abstract}
We present in this paper a MHD modelling of the gliding arc behaviour of a DC plasma torch operating with air under low current and high voltage conditions. The low current leads to instabilities and difficulties to simulate the process because the magnetic field is not sufficient to constrict the arc. The model is 3D, time-dependent and the MHD equations are solved in using CFD software Code_Saturne ${ }^{\circledR}$. Although the arc is definitively non-LTE, the LTE assumption is considered as a first approach. The injection of air is tangential. A hot gas channel reattachment model has been used to simulate the restriking process of the arc root. After the description of the model, the most appropriate electrical voltage breakdown parameter has been selected in comparing with experimental results. A typical operating point is then studied in details and shows the helical shape of the arc discharge in the nozzle. Finally, the mass flow rate and the current have been varied in the range $0.16-0.5 \mathrm{gs}^{-1}$ and 100 $-300 \mathrm{~mA}$, respectively, corresponding to typical glidarc operating points of our experimental plasma torch. The model shows good consistency with experimental data in terms of: global behaviour, arc length, mean voltage and glidarc frequency.
\end{abstract}

\section{Introduction}

The non-thermal atmospheric pressure discharges take more and more interest due to their potential applications in many domains such as: hydrogen production [1], gas treatment, surface finishing, ozone generation [2], etc. In order to improve the processes, in scope to industrialization, the physical and chemical mechanisms occurring in this type of torch have to be understood. These phenomena are complex and the literature on magnetohydrodynamic (MHD) modelling of atmospheric pressure direct current (DC) plasma torch operating at low current is very poor. At low current, the magnetic field is very low and does not constrict sufficiently the arc column to stabilize it and leads to physical instabilities which are difficult to simulate.

For several years, the Center for Energy and Processes (CEP) study the plasma-assisted conversion of heavy hydrocarbons like diesel fuel [3], gasoline [4], ethanol [5], both experimentally and numerically [6-8]. More recently, the CEP has developed a non-reactive MHD model of his low current - high voltage rotating arc reactor to investigate the quasi-continuous behaviour of the arc discharge operating with air [9]. However, in rotating arc reactors, the arc behaviour can be similar to a gliding arc reactor [10] in certain conditions of current and mass flow rate due to the stretching of the arc column.

The glidarc is characterized by the restriking of the arc.

It often, but not always, corresponds to a shortening of the arc: the distance between the anode and the cathodic arc root becomes shorter and thermodynamically more favourable. This phenomenon appears when the arc voltage reaches a threshold set by the intrinsic characteristics of: the arc, the flow, the

\footnotetext{
${ }^{4}$ To whom correspondence should be addressed: laurent.fulcheri@mines-paristech.fr
} 
geometry and/or the power source. To mimic this behaviour, the restrike mode is generally simulated by a short circuit model. Different models have been proposed to simulate the short circuit. Trelles et al [11] have developed a conducting channel reattachment model and Baudry et al [12-14] a hot gas reattachment model. Both models are based on the overcome of a specific value of the electric field, in the direction normal to the anode surface in the first model, and in the arc fringes in the second model. Trelles et al imposes a high electrical conductivity in a cylindrical channel which connects the arc to the anode surface. The second model will be detailed in the next section.

The aim of this study is to develop a numerical model of a non-thermal plasma torch under glidarc mode and to compare the main characteristics of the arc with experimental data. In the first part of this paper, the main characteristics of the model are described. In the second part, the most appropriate electric field threshold has been determined in comparing with experimental data obtained with the experimental test bench developed in ref. [3]. The results are detailed for a reference case and then the influence of the mass flow rate and the electric current are investigated.

\section{Mathematical model}

The model presented below has already been detailed in ref. [9] for the study of the quasi-continuous mode of the low current plasma torch with an axial injection. We will emphasize in this paper the main characteristics of the model. Comparing with the previous MHD model, this model has been improved with an inlet vortex profile and a restriking model.

\subsection{Assumptions}

The three-dimensional model studied is based on the following main assumptions:

- The plasma is considered as a single continuous fluid (Air) and at Local Thermodynamic Equilibrium (LTE).

- The gas is treated as incompressible and expandable. The thermodynamic properties and transport coefficients depend only on the temperature, the pressure effects can be neglected.

- The gas flow is laminar and time-dependent.

- Gravitational effects are taken into account (-x direction).

- As the torch is not water-cooled, the thermal losses are neglected at the electrodes.

The maximum Reynolds number in a cell of the torch is 2500. Inside the plasma, with the high temperatures at stake, the flow can be considered laminar. In using the method defined by Sinkevic et al. [15], the Reynolds number varies from 800 close to the inlet to 400 close to the cathode outlet. These Reynolds number values are in line with the results of Freton et al. [16].

The LTE assumption is commonly assumed for high power density plasmas. This hypothesis is a strong assumption, which has been proven to imply significant discrepancies in the arc behaviour and characteristics e.g. in the arc voltage drop [11]. For low current plasma, the non-equilibrium effects are even more significant and this hypothesis is generally not correct. Nevertheless, this assumption has been considered in a preliminary phase to overcome the numerical issues linked with the low current modelling. The objective of this study was to get the general behaviour of the plasma under restrike mode. It would obviously be interesting to develop a more complex NLTE model to take into account the nonequilibrium effects.

\subsection{Governing equations}

Considering the above-mentioned assumptions, the time-dependent fluid conservation set of equations can be written as follows:

\section{Mass conservation}

$$
\frac{\partial \rho}{\partial t}+\vec{\nabla} \cdot(\rho \vec{v})=0
$$

where $\rho$ and $\vec{v}$ are the mass density and the velocity vector.

\section{Energy conservation equation}




$$
\frac{\partial \rho h}{\partial t}+\vec{\nabla} \cdot \rho h \vec{v}-\vec{\nabla} \cdot \frac{\lambda}{C_{p}} \vec{\nabla} h=\vec{J} \cdot \vec{E}-S_{r a d}
$$

where $h, \lambda, C_{p}, \vec{J}, \vec{E}$ and $\mathrm{S}_{\mathrm{rad}}$ are the gas enthalpy, the thermal conductivity, the specific heat, the current density vector, the electric field vector and the radiation losses, respectively. The term $\mathrm{S}_{\mathrm{rad}}$ is a radiation source term depending only on temperature and calculated from the net emission coefficient at atmospheric pressure taken from ref. [17].

\section{Momentum equation}

$$
\frac{\partial \rho \vec{v}}{\partial t}+\vec{\nabla} \cdot(\rho \vec{v} \otimes \vec{v})=-\vec{\nabla} p+\vec{\nabla} \cdot \vec{\tau}+\vec{J} \times \vec{B}+\rho \vec{g}
$$

Where $\vec{\tau}, \vec{B}$ and $\vec{g}$ are the shear stress tensor, the magnetic field vector and the gravity acceleration.

The term $\vec{J} \times \vec{B}$ represents the Lorentz' forces acting on the flow. Assuming the Ohm's law approximation and the global neutrality, the set of Maxwell's equations is defined by the following equations:

$$
\begin{gathered}
\vec{J}=\sigma \vec{E} \\
\vec{E}=-\vec{\nabla} \varphi \\
-\vec{\nabla} \cdot(\sigma \vec{\nabla} \varphi)=0 \\
\vec{B}=\vec{\nabla} \times \overrightarrow{\mathrm{A}} \\
-\vec{\nabla} \cdot(\vec{\nabla} \vec{A})=\mu_{0} \overrightarrow{\mathrm{J}}
\end{gathered}
$$

where $\sigma, \overrightarrow{\mathrm{A}}$ and $\mu_{0}$ are the electrical conductivity, the vector potential and the vacuum permeability, respectively. $h, \rho, C_{p}, \sigma, \lambda$ and the dynamic viscosity $\mu$ for air depend on temperature and are taken from ref. [18] at atmospheric pressure. The pressure variations in the torch are lower than $150 \mathrm{~Pa}$ around the atmospheric pressure, so the gas flow is considered incompressible and the pressure effects can be neglected without introducing significant errors.

The coupled Navier-Stokes and Maxwell's equations are solved using the CFD software Code_Saturne ${ }^{\circledR}$ V2.0.1 [19], developed by EDF R\&D and based on the SIMPLEC algorithm and a fully implicit solution of equations. The time step is set to $10 \mu \mathrm{s}$. The calculation have been realized on a Dell PowerEdge R510 server for 3000 time steps corresponding to a numerical time of $30 \mathrm{~ms}$. The calculation lasts about 30 hours on 4 processors.

\subsection{Computational domain and boundary conditions}

The calculation domain is based on an experimental DC plasma torch composed of a plasma zone and a postdischarge zone. The schematic of the plasma torch is shown of figure 1 and the mesh grid on figure 2. Usually, in DC plasma torches, the tip electrode is the cathode. In our case, the system operates under inverse polarity. Indeed, previous works that demonstrated this configuration avoids the fast destruction of the tip electrode in low-current systems [6]. In consequence, the tip electrode is the anode where a positive high voltage is applied and the nozzle is a grounded cathode. The grid mesh, realized in using SALOME 5.1.4 [20], and contains 339000 hexa-cells and is refined at the centre of the torch and near the electrodes. The mean axial and radial spacing grid is 0.7 and $0.21 \mathrm{~mm}$, respectively. The boundary conditions are detailed in table 1 . The voltage drop in the sheath is not included in the model because it is difficult to estimate a voltage drop for the low current arc (generally between 3 and $10 \mathrm{~V}$ for high current arcs). Hence, the total voltage drop results in the voltage drop in the arc column.

In the experimental setup, the vortex is created by 2 tangential tubes. At the inlet, we only consider the post-injection zone because with the real geometry the results showed that the vorticity of the flow 
was too strong and leads to a very disturbed plasma arc and the extinction of the arc. A vortex has been implemented in the post-injection zone with an axisymetrical parabolic profile. The ratio between tangential and axial velocities is set to 0.4 in the whole study which is the maximum vorticity allowing to obtain the convergence of the model. Experimentally, the ratio is close to 1.5.

An initial hot channel is defined at the first time step with a low inflow for 50 iterations. Then, a velocity slope is applied for the following 150 time steps to reach the nominal mass flow.

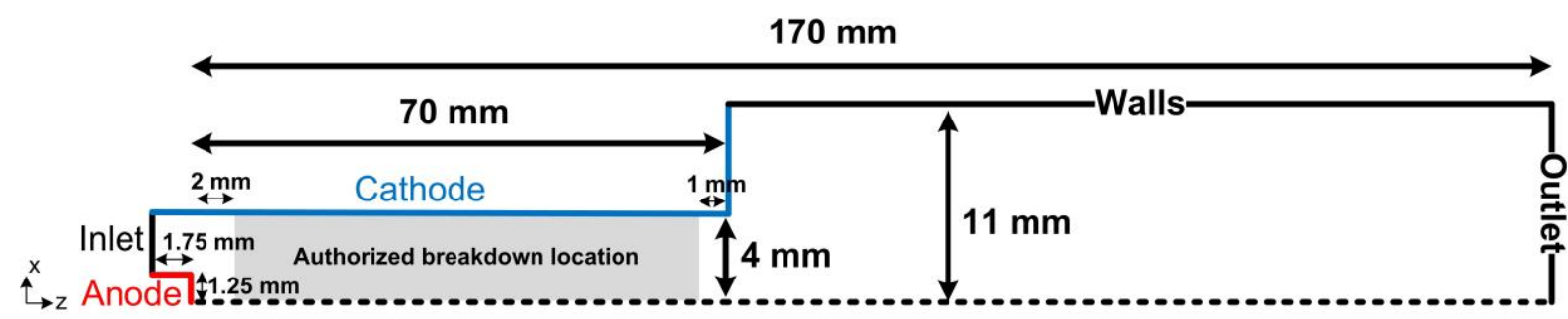

Figure 1. Schematic of the plasma torch.
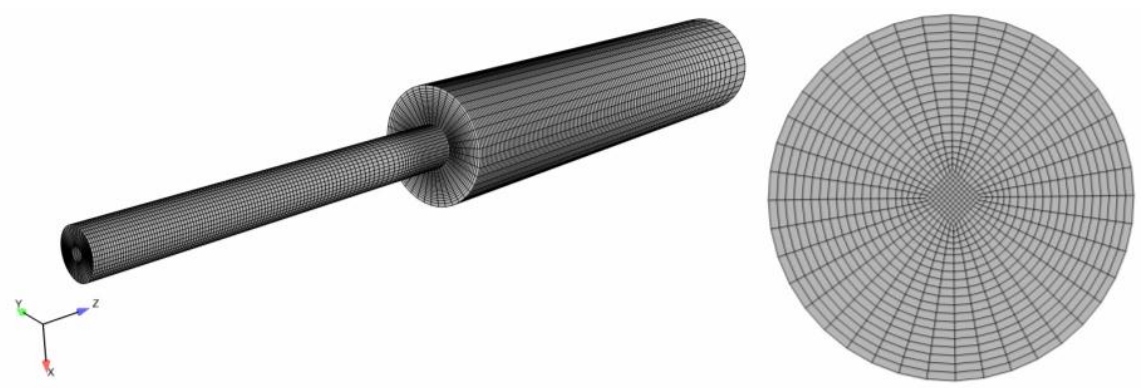

Figure 2. Computational grid of the plasma torch.

Table 1. Boundary conditions of the 3D MHD model.

\begin{tabular}{cccccc}
\hline & Inlet & Outlet & Walls & Anode & Cathode \\
\hline $\mathrm{V}_{\mathrm{i}}\left(\mathrm{ms}^{-1}\right)$ & Vortex profile & $\frac{\partial V_{i}}{\partial \vec{n}}=0$ & 0 & 0 & 0 \\
$T(\mathrm{~K})$ & 600 & $\frac{\partial T}{\partial \vec{n}}=0$ & $\frac{\partial T}{\partial \vec{n}}=0$ & $\frac{\partial T}{\partial \vec{n}}=0$ & $\frac{\partial T}{\partial \vec{n}}=0$ \\
$\varphi(\mathrm{V})$ & $\frac{\partial \varphi}{\partial \vec{n}}=0$ & $\frac{\partial \varphi}{\partial \vec{n}}=0$ & $\frac{\partial \varphi}{\partial \vec{n}}=0$ & $\varphi^{(\mathrm{n})}$ & 0 \\
$p(\mathrm{~Pa})$ & $\frac{\partial p}{\partial z}=0$ & $1.013 \times 10^{5}$ & $\frac{\partial p}{\partial \vec{n}}=0$ & $\frac{\partial p}{\partial \vec{n}}=0$ & $\frac{\partial p}{\partial \vec{n}}=0$ \\
$\mathrm{~A}_{\mathrm{i}}(\mathrm{T} . \mathrm{m})$ & $\frac{\partial A_{i}}{\partial \vec{n}}=0$ & 0 & $\frac{\partial A_{i}}{\partial \vec{n}}=0$ & $\frac{\partial A_{i}}{\partial \vec{n}}=0$ & $\frac{\partial A_{i}}{\partial \vec{n}}=0$ \\
\hline
\end{tabular}

\subsection{Arc reattachment model}

We have used for this study the hot gas column reattachment model developed by Baudry et al [1214]. The stretching of the arc column leads to an increase of the voltage drop, and therefore the electric field, between the arc column fringes and the cathode surface. The breakdown occurs when the electric field overcomes a specified threshold value of the electric field, named $\mathrm{E}_{\mathrm{b}}$, in a cell of the computational mesh. If several cells are detected, the cell where the electric field is the highest is selected. When the critical value $\mathrm{E}_{\mathrm{b}}$ is reached in the boundary layer of the arc column, the boundary conditions on the cathode potential are modified to force the new arc root to connect to the closest point of the cathode. The short circuit is imposed in creating a $1.6 \mathrm{~mm}$ diameter $6000 \mathrm{~K}$ channel for 30 time steps between the arc column fringe and the cathode wall at the location where the electric field 
threshold value has been overcome. This temperature corresponds to an electrical conductivity of 95.4 $\mathrm{S} . \mathrm{m}^{-1}$. The parameter $\mathrm{E}_{\mathrm{b}}$ has a significant influence on both the glidarc frequency and the voltage drop amplitude [21]. In our case, the breakdown electric field has been evaluated from experimental data. In order to hale the new arc root to attach at the breakdown location, a zero potential is imposed at the cathode $0.8 \mathrm{~mm}$ around the breakdown location, and a null-flux elsewhere. The new cathodic arc root is authorized to be attached between $2 \mathrm{~mm}$ downstream the anode tip and $1 \mathrm{~mm}$ upstream the cathode exit. This allows getting the hot channel fully inside the cathode and not interfering with the current readjustment. The anodic arc root remains always attached to the centre of the anode.

\section{Results and discussion}

For this study, we have focused on the comparison with experimental data. The main parameters used to compare the model and the experiments are the mean voltage and the glidarc appearance frequency. Moreover, experimental photographs allow comparing the plasma plume length.

The study has been realized in a typical range of mass flow rate and current allowing the appearance of the glidarc behaviour of the arc. This range is showed on figure 3. The quasi-continuous regime is similar to the takeover mode of high current torch defined by a quasi-periodic fluctuation of the voltage drop and the movement of the arc root. The glidarc regime, appearing with low current plasma torches, has a similar behaviour of the restrike mode of high current plasma torches which is characterized by high voltage drop amplitude, voltage fluctuations and an unstable arc motion [21]. The arc initiates at the shortest interelectrode distance where the electric field is the highest. Then, the arc root slides on the cathode, blown by the inlet flow until it reached a certain length and extinguishes. The extinction of the arc is followed by a short circuit upstream where the arc attachment is more favourable. The filamentary domain corresponds to a streamer-like discharge remaining located close to the anode and defined by high frequency $(>2 \mathrm{kHz})$ [4].

In the parametric study, the current has been varied from 100 to $300 \mathrm{~mA}$ and the mass flow rate from 0.16 to $0.5 \mathrm{gs}^{-1}$. If not otherwise specified, the reference case is used: $\mathrm{I}=250 \mathrm{~mA}, \mathrm{Q}_{\mathrm{air}}=0.32 \mathrm{gs}^{-1}, \mathrm{E}_{\mathrm{b}}=$ $3 \mathrm{kV} \cdot \mathrm{mm}^{-1}, \mathrm{~S}=0.4$.

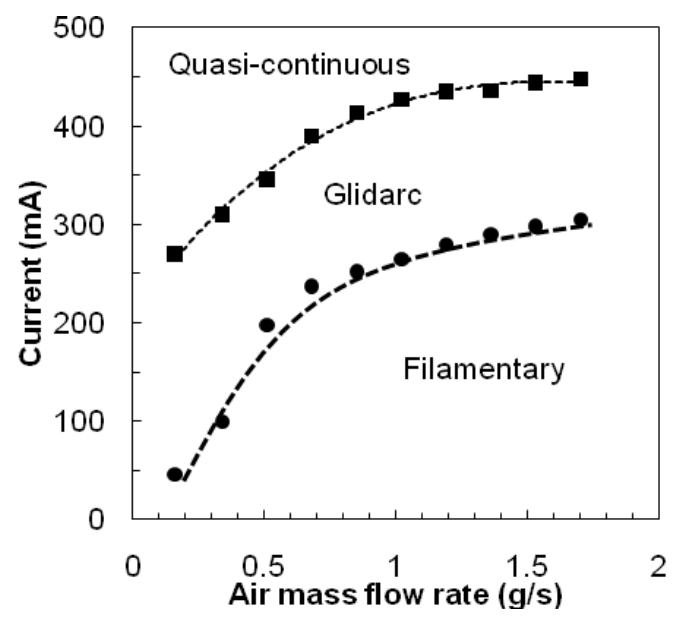

Figure 3. Arc transitions and range of study based on experimental data. (घ) Quasi-continuous glidarc transition. $(\bullet)$ Glidarc - filamentary transition.

\subsection{Determination of $E_{b}$ value}

The Paschen' law in air at atmospheric pressure and room temperature estimates the order of magnitude of $E_{b}$ at $3.6 \mathrm{kV} \cdot \mathrm{mm}^{-1}$ [22]. At $600 \mathrm{~K}$, we can estimate the breakdown voltage to $2.5 \mathrm{kV} . \mathrm{mm}^{-}$ 1. The Paschen' law is determined for a static system, without flow, in a plate-plate configuration in a dry air. In a real case, the breakdown voltage can vary significantly with the flow rate, the geometry, the humidity and the electrodes. In order to determine the most appropriate $\mathrm{E}_{\mathrm{b}}$ parameter, an investigation has been realized on this parameter and then compared to the experimental data. For $\mathrm{I}=$ $250 \mathrm{~mA}$ and $\mathrm{Q}_{\text {air }}=0.32 \mathrm{gs}^{-1}$, the experimental mean voltage is $1550 \mathrm{~V}$ and the glidarc frequency 156 
$\mathrm{Hz}$. We can observe on figure 4 and figure 5 that an $\mathrm{E}_{\mathrm{b}}$ value of $3.10^{6} \mathrm{~V} \cdot \mathrm{m}^{-1}$ is the most appropriate for our study with a mean voltage drop of $1600 \mathrm{~V}$ and a glidarc frequency of $202 \mathrm{~Hz}$. This value of $\mathrm{E}_{\mathrm{b}}$ will be used in the rest of the paper. The value of $E_{b}$ can probably be dependent of the mesh quality and will be investigated in a further study. At this stage of development, we cannot match the exact glidarc frequency. This is probably a consequence of the non-equilibrium effects and the absence of anodic and cathodic sheaths.

We can observe on both figures that the higher the $\mathrm{E}_{\mathrm{b}}$, the higher the mean voltage, but the glidarc frequency passes through a minimum value at $E_{b}=3 \mathrm{kV} \cdot \mathrm{mm}^{-1}$. Indeed, below $3 \mathrm{kV} \cdot \mathrm{mm}^{-1}$, the higher $E_{b}$, the lower the glidarc frequency, because the arc needs more time to reach the critical electric field value. Above $3 \mathrm{kV} \cdot \mathrm{mm}^{-1}$, another phenomenon appears. The new arc root attachment is more downstream as shown on figure 6 . The breakdown no longer appears close to the tip electrode but in the middle of the nozzle. As a result, the voltage drop is lower, the new breakdown appears sooner, and the mean voltage drop is higher. This highlights the fact that the arc attachment is not necessarily appearing at the shortest interelectrode distance but can depend on the arc shape or charge accumulation, for example.

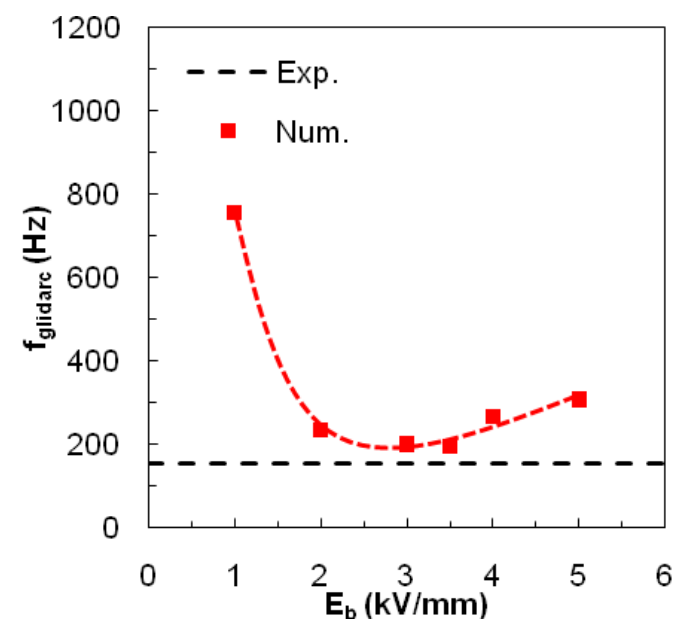

Figure 4. Influence of $E_{b}$ on glidarc frequency. $I=250 \mathrm{~mA} . Q_{a i r}=0.32$ g.s ${ }^{-1}$.

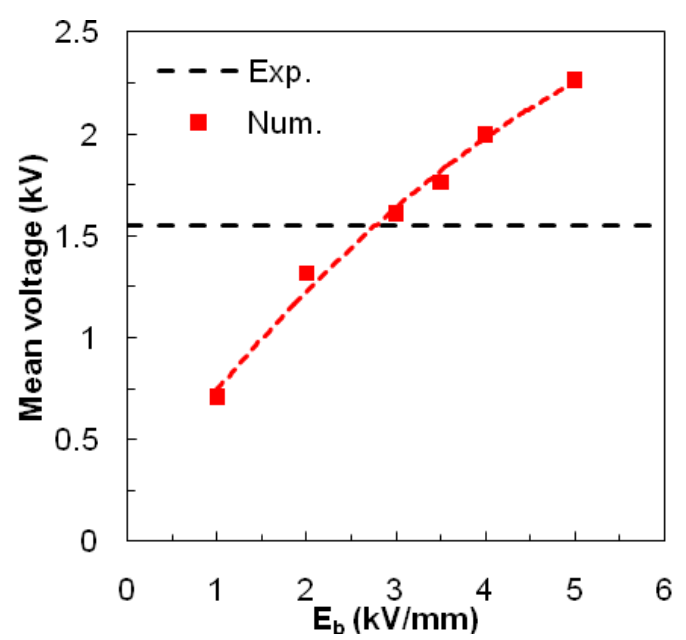

Figure 5. Influence of $E_{b}$ on mean voltage drop. 


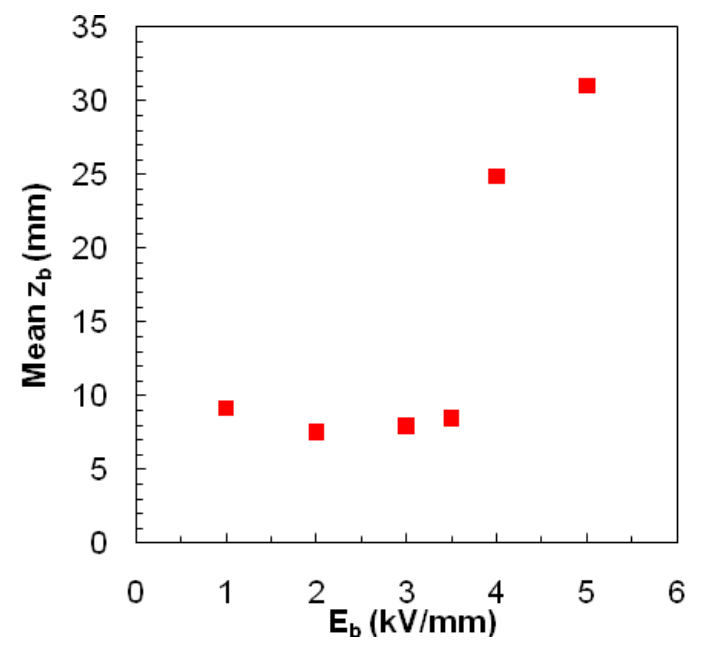

Figure 6. Mean axial position of the new arc root vs. $E_{b}$.

3.2. Results for the reference case: $I=250 \mathrm{~mA}, Q_{a i r}=0.32 \mathrm{gs}^{-1}, E_{b}=3 \mathrm{kV} . \mathrm{mm}^{-1}$.

This section shows results for a typical operating point and the comparison with quantitative and qualitative experimental results. This section points also out the main differences between the vortex and axial injection developed in a previous model [9].

Figure 7 shows the experimental and numerical oscillograms for the reference case. The deposited power is $400 \mathrm{~W}$. We can notice that the numerical glidarc frequency is slightly higher than in the experimental case, as seen in the previous section. The voltage shapes are similar for the experimental and numerical data with instabilities during the stretching of the arc and a very sharp voltage drop when the arc breaks.

Figure 8 shows a photograph taken with a fast video camera and figure 9 shows the arc shape for an iso-temperature of $3000 \mathrm{~K}$. Before breaking up, the plasma arc is attached to the cathode tip and the plasma plume length is few centimetres long. The model shows good consistency with the experimental data. We can also notice the helical shape of the arc column in the nozzle. This phenomenon has been confirmed by fast camera video (cf. figure 8 , right side). In the nozzle, the magnetic field is very low $(<1 \mathrm{mT})$ and does not constrict enough the arc column as in the high current DC plasma torch which are usually very stable. Thus, the arc column follows the vortex flow shape in the nozzle and leads to the helical shape. The rotating frequency of the arc column, based on the fast Fourier transform (FFT) of the oscillogram, is $1.5 \mathrm{kHz}$.

We do not observe on figure 10 the rotation of the arc root at the cathode tip as it is the case experimentally. After the new arc root appears, the arc root slides along the cathode (the axial position being highly correlated with the voltage) in a close radial plane. This is probably due to the lower swirl intensity applied at the inlet of the numerical model (around 1.5 experimentally), because above 0.5 , the model convergence cannot be sustained. Thus, the model is not fully predictive yet due to the limitation of the swirl value in the model. However, the results have shown that the model provides fair correlation with experiments. With the further developments of the model, it could be possible to increase the swirl value.

The electric field distribution is shown on figure 11 before the arc breakdown. One observes that the electric field is higher than $3 \mathrm{kV} \cdot \mathrm{mm}^{-1}$ before breakdown close to the anode where the tip effect is the highest. However, the arc is not allowed to break at this location to not influence the z-normal plane where the current is calculated $(z=2 \mathrm{~mm})$. After the breakdown occurring, we observe on figure 12 the $6000 \mathrm{~K}$ hot channel which connects the fringes of the arc column to the cathode. The previous arc is extinguished quickly.

The additional energy imposed by the hot channel can be estimated to only $0.4 \%$ on one period of the total energy dissipated by the plasma. Therefore, this low amount of energy does not disturb significantly the hydrodynamic of the arc. 


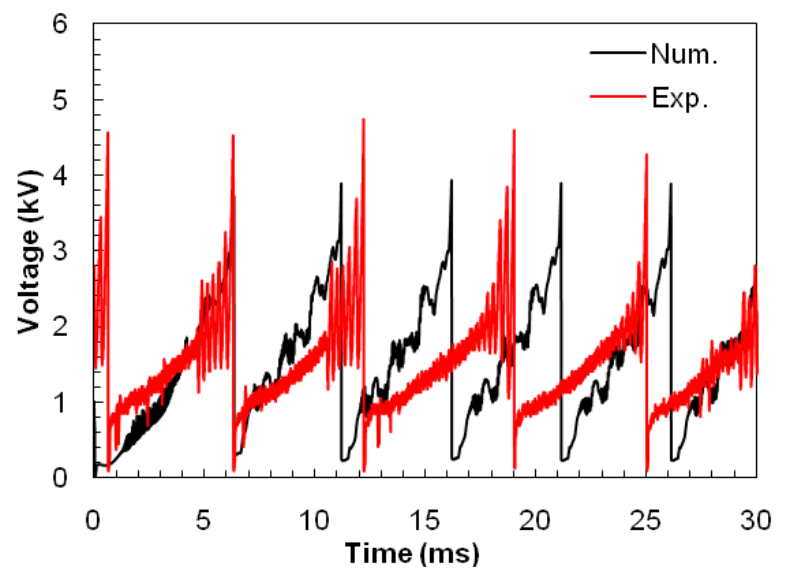

Figure 7. Experimental and numerical voltage vs. time. $\mathrm{I}=0.25 \mathrm{~A} . \mathrm{Q}_{\mathrm{air}}=0.32 \mathrm{gs}^{-1}$.

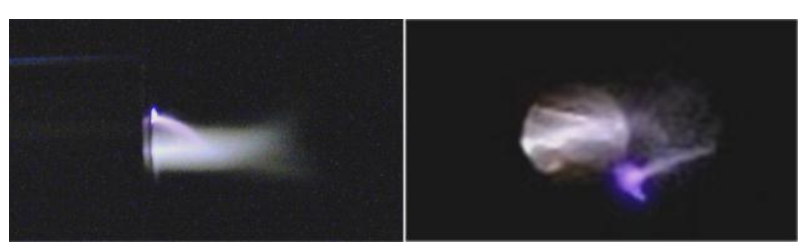

Figure 8. Fast camera photograph of the plasma arc at the tip of the nozzle. Left: side view. Right: Front view. $\mathrm{I}=0.25 \mathrm{~A}$. $\mathrm{Q}_{\text {air }}=0.32 \mathrm{gs}^{-1}$.

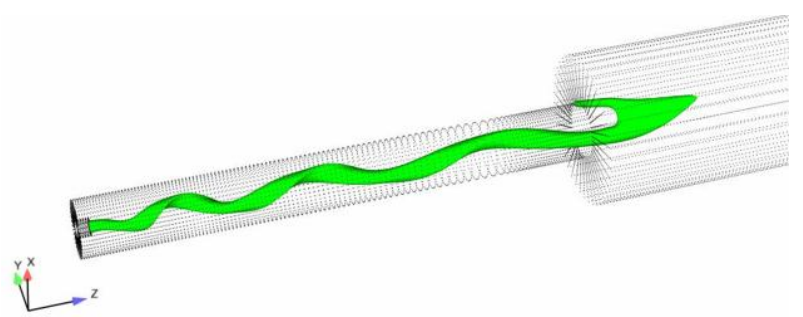

Figure 9. Iso-temperature $(3000 \mathrm{~K})$ just before breakdown. $\mathrm{t}=16 \mathrm{~ms} . \mathrm{I}=0.25 \mathrm{~A} . \mathrm{Q}_{\text {air }}=0.32 \mathrm{gs}^{-1}$.

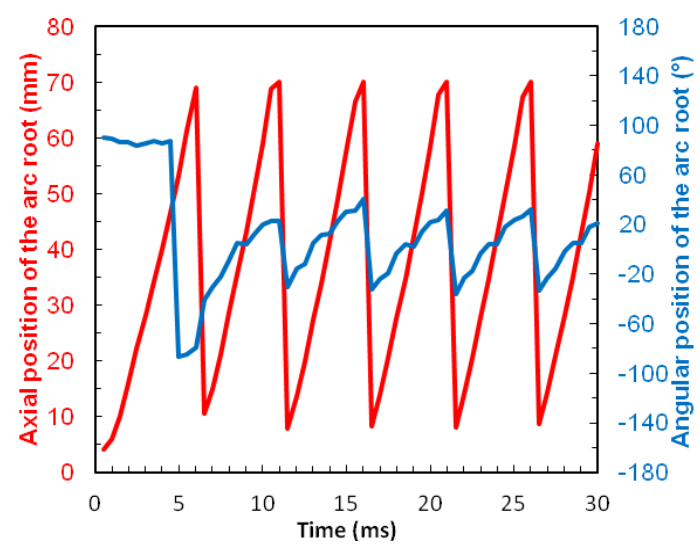

Figure 10. Axial and angular position of the cathodic arc root 


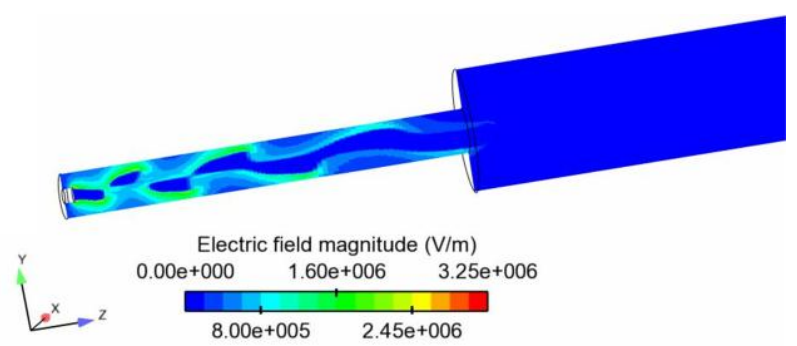

Figure 11. Electric field distribution before breakdown in a radial cross section.

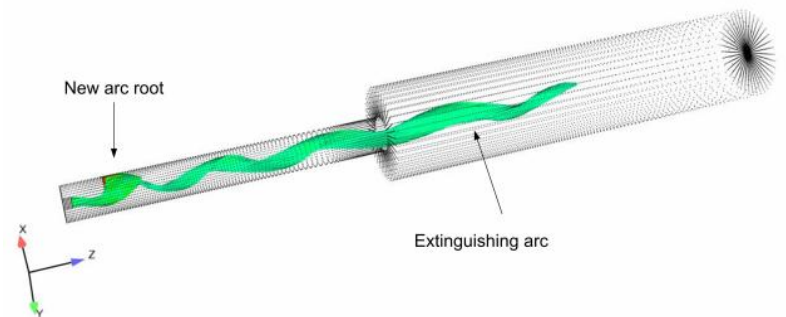

Figure 12. Iso-volume ( 2000 to $6000 \mathrm{~K}$ ) coloured by temperature showing a new arc attachment just after the 30 time steps where the hot channel is imposed.

\subsection{Influence of the current}

The influence of the low current applied is shown on figure 13 and figure 14 in terms of glidarc frequency and mean voltage drop. Concerning the glidarc frequency, the results are very close from experimental data and the trends are similar under $250 \mathrm{~mA}$. For $300 \mathrm{~mA}$, the point does not follow the experimental trend. For this specific point, the mean axial position of breakdown is higher than the other cases $(13 \mathrm{~mm})$. We can observe experimentally that the higher the current, the lower the glidarc frequency. For $\mathrm{I}=300 \mathrm{~mA}$, the experimental glidarc frequency is very low because we are close to the glidarc - quasi-continuous transition. The model shows a fair estimation of the mean voltage drop.

On figure 15, the experimental photographs show that under $200 \mathrm{~mA}$, the arc plume stays in the nozzle. For $I=200 \mathrm{~mA}$, the arc plume goes out slightly like in the model and over $200 \mathrm{~mA}$, the arc plume is around $20 \mathrm{~mm}$ long. The model estimates pretty well the arc length. For $\mathrm{I}=300 \mathrm{~mA}$, we observe experimentally that there is a unique arc root but several streamer-like arcs connecting the arc root to the arc column, which cannot be observed in the model.

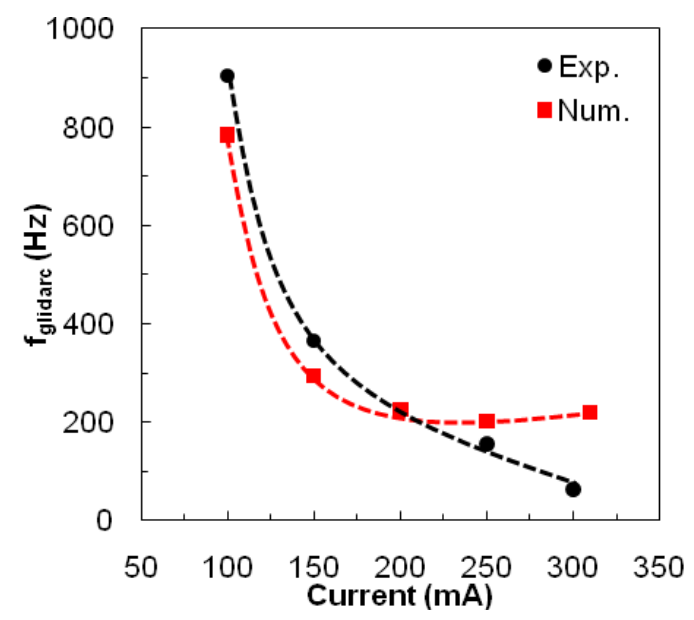

Figure 13. Influence of the electrical current on the glidarc frequency. $Q_{\text {air }}=0.32 \mathrm{gs}^{-1}$. 


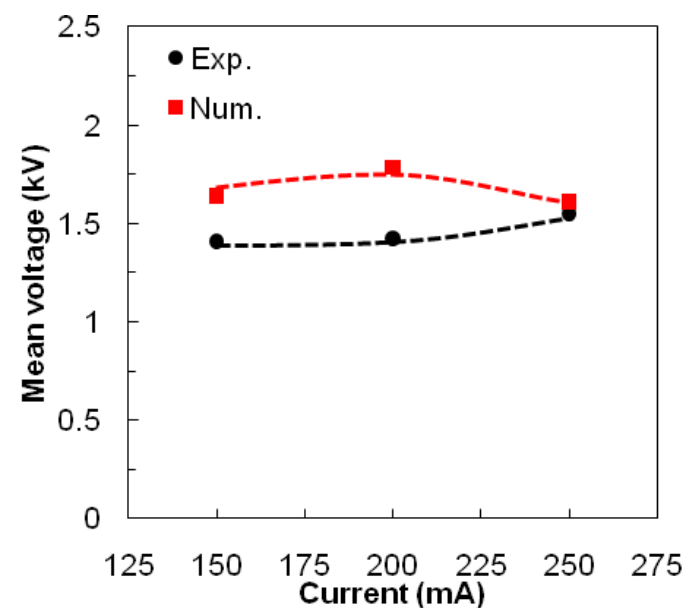

Figure 14. Influence of the current on the mean voltage drop. $\mathrm{Q}_{\mathrm{air}}=0.32 \mathrm{gs}^{-1}$.

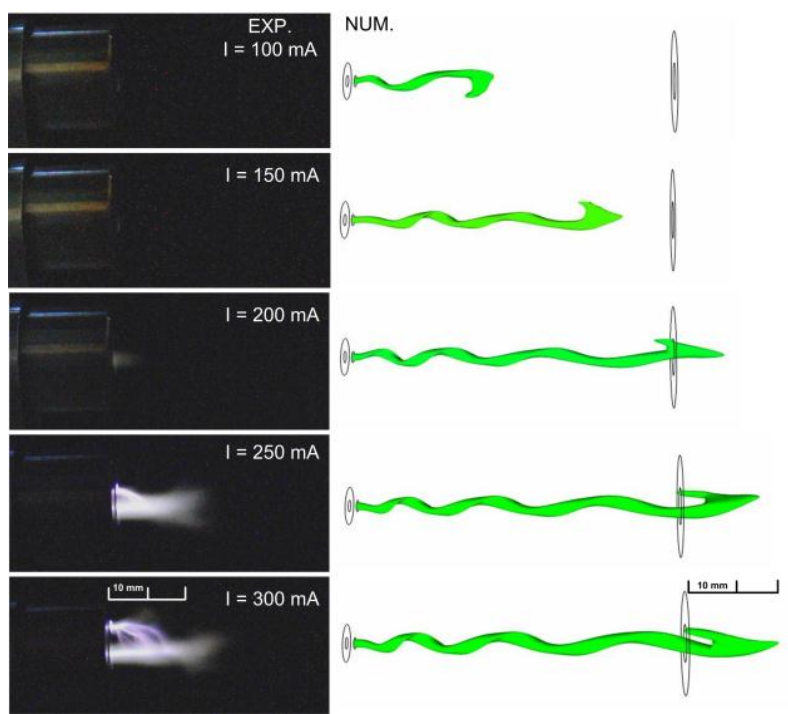

Figure 15. Photographs of the plasma plume at the nozzle tip (left) and corresponding numerical plasma length (right) at the time step before breakdown in function of current.

\subsection{Influence of the mass flow rate}

Figure 16 shows fair trend correlation of the glidarc frequency for a mass flow rate between 0.29 and $0.4 \mathrm{gs}^{-1}$. Under $0.29 \mathrm{gs}^{-1}$, the arc no longer breaks because the $\mathrm{E}_{\mathrm{b}}$ parameter is probably overestimated on this range. Over $0.29 \mathrm{gs}^{-1}$, the arc breaks close to the anode and the higher the air mass flow rate, the higher the glidarc frequency. The flow stretches the arc quicker, and the critical is reached sooner leading to the increase of the frequency. Over $0.4 \mathrm{gs}^{-1}$, the experimental data tends to the filamentary regime defined by other frequencies. However, on figure 17, we can observe that the model matches well the mean voltage drop in the whole range. 


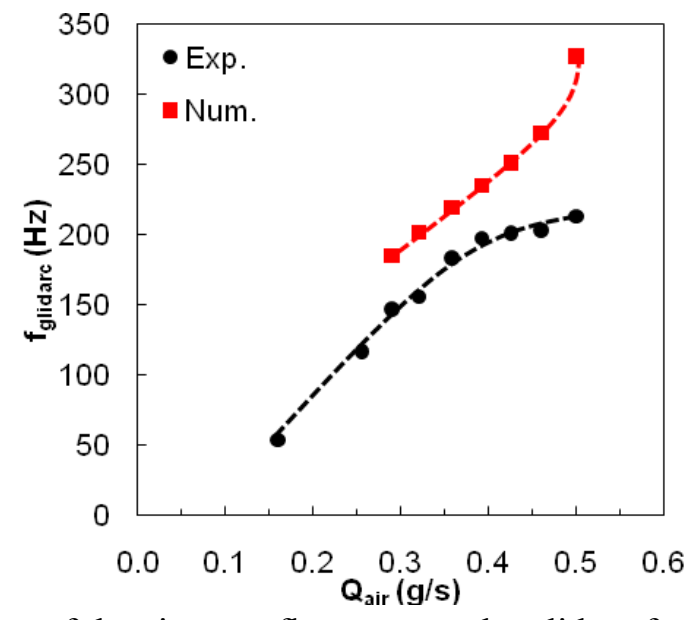

Figure 16. Influence of the air mass flow rate on the glidarc frequency. $\mathrm{I}=250 \mathrm{~mA}$.

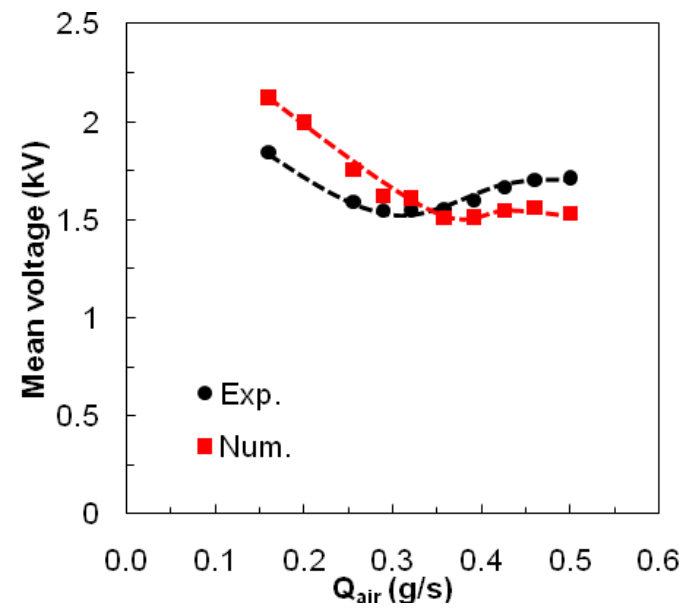

Figure 17. Influence of the air mass flow rate on the mean voltage drop. $I=250 \mathrm{~mA}$.

Looking at the mean voltage trend $v s$. mass flow rate, we can infer that the plasma plume length decreases with the mass flow rate. This is confirmed by experimental photographs shown on figure 18 . This phenomenon is well-known and is due to the Reynolds number which increases with the flow velocity. The laminar plasma discharges are usually longer than transient or turbulent ones. We also observe that for low mass flow rates, the plasma column is very stable does not have a helical shape.
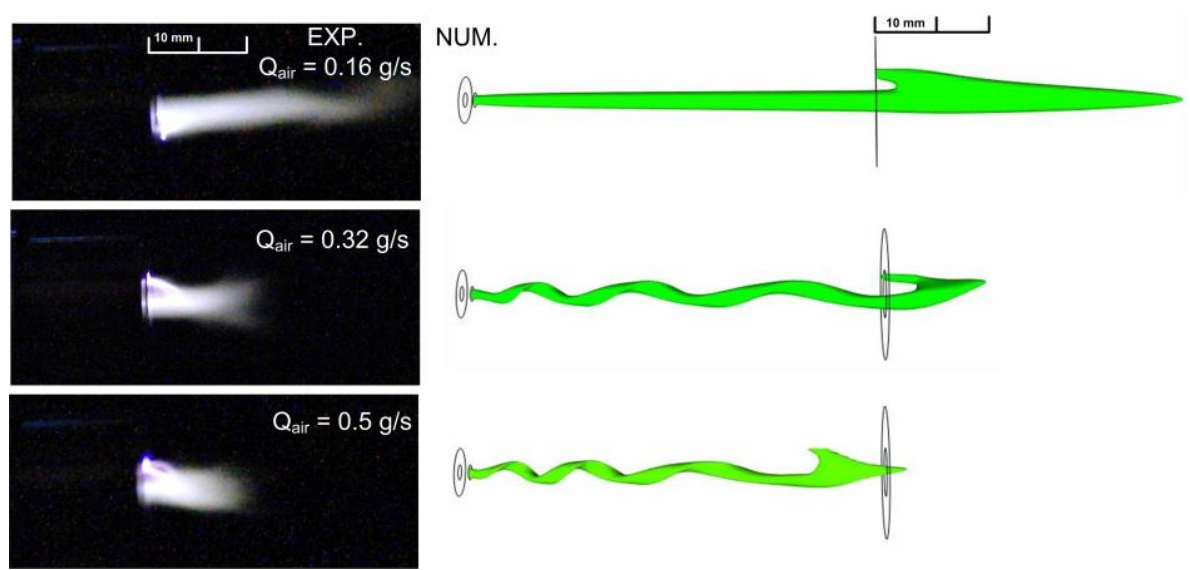

Figure 18. Photographs of the plasma plume at the nozzle tip (left) and corresponding plasma length (right) at the time step before breakdown in function of the air mass flow rate. 
Even if the LTE is assumed, the model shows good agreement because the $\mathrm{E}_{\mathrm{b}}$ parameter is calibrated on experimental data. With a non-equilibrium model, this value of $\mathrm{E}_{\mathrm{b}}$ will probably be different. Thus, the $\mathrm{E}_{\mathrm{b}}$ parameter can be used as a « fitting » parameter.

\section{Conclusions and perspectives}

A previous MHD model operating with a very low current has been modified to take into account the inlet vortex profile and the restrike mode behaviour. First, a critical breakdown electric field of 3 $\mathrm{kV} \cdot \mathrm{mm}^{-1}$ supplies good correlation with experimental data in terms of mean voltage drop and glidarc frequency. For a reference case $\left(\mathrm{I}=250 \mathrm{~mA}, \mathrm{Q}_{\mathrm{air}}=0.32 \mathrm{gs}^{-1}\right)$, the results show a helical shape of the arc column due to the vortex injection. The rotation frequency of the arc column is around $1500 \mathrm{~Hz}$ and leads to instabilities in the numerical voltage oscillograms. Fast camera photograph have confirmed the helical shape of the arc column inside the nozzle. The magnetic field is too low to constrict the arc column which follows the flow shape. The model estimates well the plasma plume length.

Then, a parametric study has been realized on current and mass flow rate and shows fair correlation with experimental data for the predicted glidarc frequency, mean voltage drop and arc length.

In a further stage of development of our model, a non-LTE will be considered to simulate the nonequilibrium effects in low current DC plasma torches. It has been demonstrated by Trelles et al that with a non-LTE, no reattachment process is needed to simulate the steady and/or takeover mode [11] but it will be required for the restrike mode to get more accurate results [21].

\section{Acknowledgments}

The authors gratefully acknowledge Renault for their financial support and EDF R\&D for their technical support.

\section{References}

[1] Petitpas G, Rollier J D, Darmon A, Gonzalez-Aguilar J, Metkemeijer R and Fulcheri L 2007 A comparative study of non-thermal plasma assisted reforming technologies Int. J. Hydrogen Energy 32 2848-67

[2] Fridman A, Chirokov A and Gutsol A 2005 Non-thermal atmospheric pressure discharges $J$. Phys. D, Appl. Phys. $38 \mathrm{R} 1$

[3] Lebouvier A, Fresnet F, Fabry F, Boch V, Rohani V, Cauneau F and Fulcheri L 2011 Exhaust gas fuel reforming of Diesel fuel by non-thermal arc discharge for NOx trap regeneration application Energy Fuels 25 1034-44

[4] Rollier J D, Gonzalez-Aguilar J, Petitpas G, Darmon A, Fulcheri L and Metkemeijer R 2008 Experimental Study on Gasoline Reforming Assisted by Nonthermal Arc Discharge Energy Fuels 22 $556-60$

[5] Petitpas G, Gonzalez-Aguilar J, Darmon A and Fulcheri L 2010 Ethanol and E85 reforming assisted by a non-thermal arc discharge Energy Fuels 24 2607-13

[6] Rollier J D, Petitpas G, Gonzalez-Aguilar J, Darmon A, Fulcheri L and R. Metkemeijer 2008 Thermodynamics and kinetics analysis of gasoline reforming assisted by arc discharge Energy Fuels 22 1888-93

[7] Gonzalez-Aguilar J, Petitpas G, Lebouvier A, Rollier J D, Darmon A and Fulcheri L 2009 Three stages modeling of n-octane reforming assisted by a nonthermal arc discharge Energy Fuels 23 49316

[8] Lebouvier A, Cauneau F and Fulcheri L 2011 2D axisymmetric coupled computational fluid dynamics-kinetics modeling of a nonthermal arc plasma torch for diesel fuel reforming Energy Fuels accepted for publication

[9] Lebouvier A, Delalondre C, Fresnet F, Boch V, Rohani V, Cauneau F and Fulcheri L 2011 Threedimensional unsteady MHD modeling of a low current - high voltage non-transferred DC plasma torch Operating with air IEEE Trans. Plasma Sci. 39 1889-99

[10] Czernichowski A 1994 Gliding arc - Applications to engineering and environment control Pure Appl. Chem. 66 1301-10 
[11] Trelles J P, Heberlein J V R and Pfender E 2007 Non-equilibrium modelling of arc plasma torches J. Phys. D, Appl. Phys. 40 5937-52

[12] Moreau E, Chazelas C, Mariaux G and Vardelle A 2006 Modeling the restrike mode operation of a DC plasma spray torch $J$. Therm. Spray Tech. 15 524-30

[13] Baudry C, Vardelle A and Mariaux G 2005 Numerical modeling of a DC non-transferred plasma torch: Movement of the arc anode attachment and resulting anode erosion High Temp. Mat. Proc. $91-$ 15

[14] Chazelas C, Moreau E, Mariaux G and Vardelle A 2006 Numerical modelling of arc behaviour in a DC plasma torch High Temp. Mat. Proc. 10 393-406

[15] Sinkevich O A, Chikunov S E, Glazkov V V and Isakaev E Kh 2002 Boundaries of stability of the plasma flow in cylindrical channels TPP 7 Thermal Plasma Processes (Strasbourg, France) (Under press in the Progress in Plasma Processing of Materials 2002 published by Begell house, Inc, New York)

[16] Freton P, Gonzalez J J, Camy Peyret F and Gleizes A 2003 Complementary experimental and theoretical approaches to the determination of the plasma characteristics in a cutting plasma torch $J$. Phys. D, Appl. Phys. 36 1269-83

[17] Naghizadeh-Kashani Y, Cressault Y and Gleizes A 2002 Net emission coefficient of air thermal plasmas J. Phys. D, Appl. Phys. 35 2925-34

[18] D'Angola A, Colonna G, Gorse C and Capitelli M 2008 Thermodynamic and transport properties in equilibrium air plasmas in a wide pressure and temperature range Eur. Phys. J. D 46 129-50

[19] Archambeau F, Mechitoua N and Sakiz M, 2004 Code Saturne: a finite volume code for the computation of turbulent incompressible flows International Journal on Finite Volumes $\mathbf{1}$

[20] http://www.salome-platform.org/

[21] Trelles J P, Chazelas C, Vardelle A and Heberlein J V R 2009 Arc Plasma Torch Modeling J. Therm. Spray Tech. 18 728-52

[22] Naidu M S and Kamaraju V 1995 High voltage engineering, $2^{\text {nd }}$ Edition, MacGraw Hill 